\title{
Maximizing Adherence and Gaining New Information For Your Chronic Obstructive Pulmonary Disease (MAGNIFY COPD): Study Protocol for the Pragmatic, Cluster Randomized Trial Evaluating the Impact of Dual Bronchodilator with Add-On Sensor and Electronic Monitoring on Clinical Outcomes
}

\author{
David Price, (iD 1,2 Rupert Jones, ${ }^{3}$ \\ Pascal Pfister, iD ${ }^{4}$ Hui Cao, ${ }^{5}$ Victoria Carter, \\ Anu Kemppinen, ${ }^{6}$ Björn Holzhauer, (iD ${ }^{4}$ \\ Alan Kaplan, ${ }^{7}$ Allan Clark, ${ }^{8}$ David MG Halpin, (D) \\ Hilary Pinnock, ${ }^{10}$ James D Chalmers, \\ Job FM van Boven, iD ${ }^{12}$ Kai M Beeh, ${ }^{13}$ \\ Konstantinos Kostikas, (ID ${ }^{14}$ Nicolas Roche, (iD ${ }^{15}$ \\ Omar Usmani, (iD ${ }^{16}$ Paul Mastoridis (iD) ${ }^{5}$ \\ 'Observational and Pragmatic Research Institute, \\ Singapore, Singapore; ${ }^{2}$ Centre of Academic Primary \\ Care, Division of Applied Health Sciences, University \\ of Aberdeen, Aberdeen, UK; ${ }^{3}$ Faculty of Health, \\ University of Plymouth, Plymouth, Devon, UK; \\ ${ }^{4}$ Novartis Pharma AG, Basel, Switzerland; ${ }^{5}$ Novartis \\ Pharmaceuticals Corporation, East Hanover, NJ, USA; \\ ${ }^{6}$ Observational and Pragmatic Research International \\ Ltd, Stubbs House Stubbs Green, London, Norwich, \\ UK; ; Department of Family and Community \\ Medicine, University of Toronto, Toronto, Ontario, \\ Canada; ${ }^{8}$ Norwich Medical School, University of East

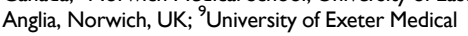 \\ School, College of Medicine and Health, University of \\ Exeter, Exeter, UK; ${ }^{10}$ Allergy and Respiratory \\ Research Group, Usher Institute, University of \\ Edinburgh, Edinburgh, UK; " College of Medicine, \\ University of Dundee, Dundee, UK; ${ }^{12}$ Department of \\ Clinical Pharmacy \& Pharmacology, Groningen \\ Research Institute for Asthma and COPD (GRIAC), \\ University Medical Center Groningen, University of \\ Groningen, Groningen, the Netherlands; ${ }^{13}$ Clinical \\ Research, Insaf Respiratory Research Institute, \\ Wiesbaden, Germany; ${ }^{14}$ Respiratory Medicine \\ Department, University of loannina School of \\ Medicine, loannina, Greece; ${ }^{15}$ Cochin Hospital and \\ Institute, APHP Centre, University of Paris, Paris, \\ France; ${ }^{16}$ National Heart \& Lung Institute (NHLI), \\ Imperial College London and Royal Brompton \\ Hospital, London, UK
}

Correspondence: David Price Centre of Academic Primary Care, Division of Applied Health Sciences, University of Aberdeen, Polwarth Building, Foresterhill, Aberdeen, AB25 2ZD, UK

Tel +44656962 3627

Email dprice@opri.sg
Background: Poor treatment adherence in COPD patients is associated with poor clinical outcomes and increased healthcare burden. Personalized approaches for adherence management, supported with technology-based interventions, may offer benefits to patients and providers but are currently unproven in terms of clinical outcomes as opposed to adherence outcomes.

Methods: Maximizing Adherence and Gaining New Information For Your COPD (MAGNIFY COPD study), a pragmatic cluster randomized trial, aims to evaluate the impact of an adherence technology package (interventional package), comprising an adherence review, ongoing provision of a dual bronchodilator but with an add-on inhaler sensor device and a connected mobile application. This will compare time to treatment failure and other clinical outcomes in patients identified at high risk of exacerbations with historic poor treatment adherence as measured by prescription collection to mono/dual therapy over one year (1312 patients) versus usual care. Treatment failure is defined as the first occurrence of one of the following: (1) moderate/severe COPD exacerbation, (2) prescription of triple therapy (inhaled corticosteroid/long-acting $\beta_{2}$ agonist/long-acting muscarinic antagonist [ICS/LABA/LAMA]), (3) prescription of additional chronic therapy for COPD, or (4) respiratory-related death. Adherence, moderate/severe exacerbations, respiratory-related healthcare resource utilization and costs, and intervention package acceptance rate will also be assessed. Eligible primary care practices $(\mathrm{N}=176)$ participating in the Optimum Patient Care Quality Improvement Program will be randomized (1:1) to either adherence support cluster arm (suitable patients already receiving or initiated Ultibro ${ }^{\circledR}$ Breezhaler $^{(}$[indacaterol/glycopyrronium] will be offered interventional package) or the control cluster arm (suitable patients continue to receive usual clinical care). Patients will be identified and outcomes collected from anonymized electronic medical records within the Optimum Patient Care Research Database. On study completion, electronic medical record data will be reextracted to analyze outcomes in both study groups.

Registration Number: ISRCTN10567920.

Conclusion: MAGNIFY will explore patient benefits of technology-based interventions for electronic adherence monitoring.

Keywords: adherence monitoring, applications, chronic obstructive pulmonary disease, medication adherence, pragmatic randomized clinical trial, technology 


\section{Introduction}

Chronic obstructive pulmonary disease (COPD) remains amongst the top eight leading causes of disease burden worldwide. ${ }^{1}$ Patients with COPD often experience exacerbations and worsening of symptoms, which may result in hospitalizations and disease progression. ${ }^{2}$ Inhaled therapies in COPD have proven to be effective in reducing symptoms, frequency and severity of exacerbations, and improving quality of life of exacerbating patients. ${ }^{3}$

Poor adherence to inhaled treatment remains, however, a significant problem in COPD patients, often associated with worse exacerbation rates and symptom control, increased healthcare resources utilization, and reduced health-related quality of life. ${ }^{2,4-7}$ Among COPD patients in a clinical setting, mean non-adherence (definition of non-adherence varied among studies) to inhaled medications ranges between $20-60 \% .^{4,8-13}$ Incorrect use and poor inhalation technique are also associated with poor disease control in such patients, ${ }^{14-16}$ with the estimated prevalence of incorrect inhalation technique ranging between 14-90\%. ${ }^{17}$ The Global Initiative for Chronic Obstructive Lung Disease (GOLD) Report emphasizes the importance of adherence and demonstration of proper inhalation technique to patients with COPD. ${ }^{18}$ The development of personalized, data-driven approaches aided by new healthcare interventions is expected to play a major role in improving medication adherence in such patients through smart inhalers, electronic reminders and monitoring. ${ }^{19,20}$ Recently, a connected inhaler system (comprising clip-on inhaler sensors, a patient-facing app, and a healthcare professional dashboard) was shown to improve adherence to maintenance therapy in patients with respiratory disease; however, such system did not show an improvement in clinical outcomes, possibly due to high adherence in the control arm. $^{21}$ This suggests the need for studies where the control arm is maintained as usual care.

Medication adherence assessment remains a challenge in clinical research because there is no consistency in the methods by which adherence is measured in COPD. ${ }^{22}$ Medication adherence rates are usually higher and overestimated in randomized clinical trials (RCTs) compared with real-world studies. ${ }^{2}$ This is due to a specific, welldefined and highly motivated population, frequent patient follow-up, and stringent adherence measures adopted in RCTs, very different to a real-life setting where most patients may not be interested in or eligible for research. ${ }^{23}$ However, even when medication is beneficial in such optimized conditions, variations in adherence are associated with major outcomes such as mortality and hospital admissions. ${ }^{24}$ Pragmatic adherence studies can mimic clinical scenarios and patients' natural behavior to evaluate whether findings from an RCT can be applied to heterogeneous populations in real life. ${ }^{23}$ Pragmatic RCTs imitate routine clinical practice and offer critical evidence for decision making by policy-makers, physicians, and patients. $^{25}$

Here, we describe the protocol of the MAGNIFY (Maximizing Adherence and Gaining New Information For Your COPD) study, an ongoing (ISRCTN10567920), pragmatic, cluster RCT. MAGNIFY is the first of its kind that aims at evaluating the impact of an adherence technology package (interventional package) on time to treatment failure and other outcomes in patients with COPD at high risk of exacerbations and with historic poor treatment adherence to mono/dual therapy over one year. The intervention package includes Ultibro ${ }^{\circledR}$ (indacaterol/glycopyrronium) Breezhaler $^{\circledR} \quad$ (Novartis, Basel, Switzerland) with a Conformité Européenne (CE) marked add-on inhaler sensor device (add-on device) and app connected mobile application. Cluster randomization was preferred to avoid cross contamination within practices and keep the trial findings relevant for real-world practice by combining real-world evidence and randomization and utilizing a heterogeneous population to inform treatment effectiveness and healthcare decisions. $^{26}$ Throughout the study, routine care will be continued, with no protocol mandated patient visits.

\section{Objectives}

The primary objective is to compare the time to treatment failure (see below for definition) between patients randomized to receive the intervention package versus those receiving usual routine clinical care (control arm). Patients will be identified based on their electronic medical records and will be randomized to the interventional package, which comprises an adherence review, ongoing provision of a dual bronchodilator but with an add-on inhaler sensor device and a connected mobile application (adherence support arm [ASA]).

The secondary objectives are to assess: (1) the proportion of patients with at least one moderate/severe exacerbation at 12 months and total number of exacerbations at 12 months based on electronic medical record data, and (2) 
adherence (based on prescription refill records) over 12 months.

The exploratory objectives are to assess: (1) respiratory-related healthcare resource utilization and costs per patient per year, defined by assessing the costs from the Optimum Patient Care Research Database, (2) the intervention package acceptance rate at the ASA sites, and (3) primary and secondary endpoints, and respiratory-related healthcare resource utilization and costs, in the secondary and exploratory populations (see Methods section for definition on different populations), and in the group of patients in the ASA versus a subgroup of patients on indacaterol/glycopyrronium Breezhaler ${ }^{\circledR}$ (standard) in the control and ASA arms.

\section{Methods Study Design}

MAGNIFY is a one-year, pragmatic, cluster randomized, multicenter, single-country trial to be run at an estimated 176 primary care practices in the UK starting January 2020. Considering the design and requirements of the study, the most efficient option was to perform the study in a setting where a single database with integrated prescribing data including refill rates and health outcomes of interest is available. This could be best obtained only in the UK, leading to design the study as a single-country trial. The primary care practice is the unit of randomization and the practices will be randomized in a 1:1 ratio to either the ASA or the control $\operatorname{arm}^{27,28}$ (Figure 1).

Anonymized electronic medical record (EMR) data will be used at baseline to identify suitable patients at each site prior to the study start. Electronic medical record data will then be extracted for defined data analyses in all sites on study completion (one year, i.e. 365 days).

To allow feasibility assessment of practices as study sites and to collect outcome data, all participating study sites will have to participate in the Optimum Patient Care (OPC) quality improvement program for COPD. ${ }^{29}$ OPC contributes data to the Optimum Patient Care Research Database (OPCRD), a UK based longitudinal electronic health record database of over 900 practices and 11 million patients with fully characterized chronic disease data. From these 900 practices, 176 practices will be selected to participate in the study based on their prescribing of the appropriate medicines to patients. The patients at practices in the ASA arm will get a full COPD quality improvement program including the OPC COPD review questionnaire and the intervention package, whereas the patients at practices in the control arm will continue to receive their usual routine clinical care. As this is a real-world study with care provided by UK physicians, the treatment will not be altered. In order to avoid any potential influence that administering a questionnaire might have on patient behavior in the control group at the beginning of the study, technology acceptance will be evaluated at the end of the 12-month study period as part of standard COPD review. Incorporating questionnaires for control arm at the beginning would also require some feedback to the clinicians. This could influence their behavior, thus compromising a true "standard of care" comparison.

\section{Trial Participants}

Primary Care Practices

Inclusion criteria include practices (1) located in the UK (England, Scotland, Wales, Northern Ireland), (2) participating in the OPC Quality Improvement programs that support chronic disease review and in addition, provide high quality respiratory data, (3) willing to use advanced technology as part of clinical practice, (4) having at least eight patients suitable for adherence technology, and (5) actively prescribing indacaterol/glycopyrronium Breezhaler ${ }^{\circledR}$. Exclusion criteria include practices not actively prescribing indacaterol/ glycopyrronium Breezhaler ${ }^{\circledR}$ to COPD patients as part of routine clinical care, and those hosting or affected by research, or other aspects of care, which might significantly influence the practice-wide implementation.

\section{Optimum Patient Care (OPC) Quality Improvement Services}

To take part in the study, all practices will have to have previously joined the OPC asthma and COPD quality improvement service (https://optimumpatientcare.org/qual ity-improvement). OPC is a social enterprise providing quality improvement since 2005 to over 900 primary care practices in the UK (about 1 in 8 practices). Pseudonymized data is remotely extracted and analyzed and reports generated providing practice level performance data and individual patient level data for recommendations for management according to national guidance.

\section{Patients}

Inclusion criteria include patients aged $\geq 40$ years, eversmokers, on the COPD register of participating practices, with coded COPD diagnosis and post-bronchodilator forced expiratory volume in 1 second $\left(\mathrm{FEV}_{1}\right)$ /forced vital capacity ever recorded $<0.7$, with at least two moderate/ 


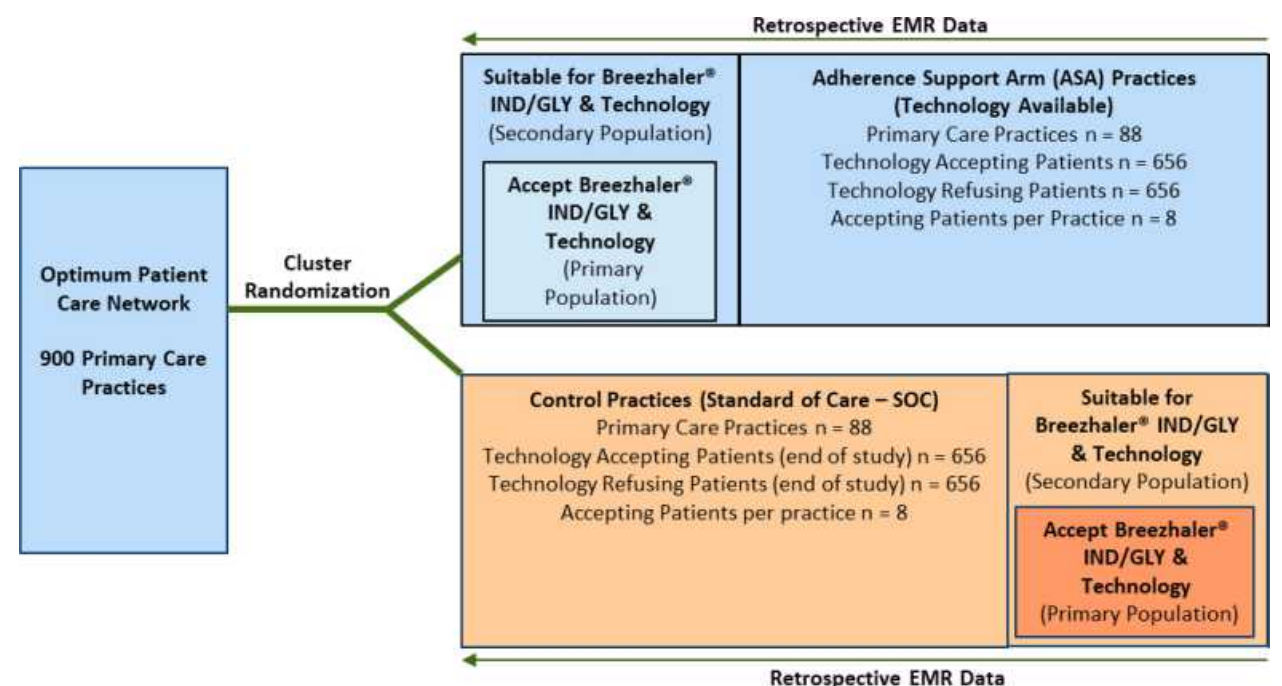

Figure I MAGNIFY trial design.

Note: It is assumed that 82 practices in each arm will complete the study.

Abbreviation: EMR, electronic medical record.

severe exacerbations in the last 24 months, and with total adherence to current mono/dual therapy of $\leq 50 \%$ based on refill pharmacy data in the last 12 months. Exclusion criteria include patients with a consent refusal code indicating opt-out of data used for research, those not deemed clinically appropriate for the therapy which is technology compatible, on triple therapy (ICS/LABA/LAMA), with read code for asthma, blood eosinophil count $>300$ cells/ $\mu \mathrm{L}$ (if blood cell count available in the prior 12 months), and those unable to use technology (not having/able to use a smartphone compatible with the technology, not having reliable internet access).

\section{Randomization}

Randomization code will be generated via a computer code and randomization will be stratified according to practice size, deprivation score and the percentage of patients with COPD with a record of $\mathrm{FEV}_{1}$ in the preceding 12 months. The data for all three stratification factors can be obtained for all National Health Service primary care practices through Public Health England (and equivalent for the devolved nations).$^{30}$ For each factor, median value from nationally available data will be used as a cutoff to categorize each factor into two strata.

\section{Intervention}

\section{Adherence Support Arm (ASA)}

In practices randomized to the ASA cluster arm, suitable patients already receiving indacaterol/glycopyrronium, or initiated on indacaterol/glycopyrronium, will be offered an intervention package, i.e. add-on device and a connected mobile application (Propeller Health, Wisconsin, USA), which have been specifically customized for indacaterol/ glycopyrronium. All sites randomized to the ASA will receive training from Propeller Health (Wisconsin, USA) on the use of the add-on device + app, for the healthcare professionals to instruct the patients on the use of this technology.

Detailed description of the intervention package can be found in the Supplementary File S1.

\section{Control Cluster Arm}

Practices randomized to the control arm will continue to provide their usual routine clinical care.

\section{Endpoints}

The primary endpoint is difference in time (in whole days) to treatment failure from baseline to the first occurrence of one of the following: (1) moderate/severe COPD exacerbation, (2) prescription of triple therapy (inhaled corticosteroid/long-acting $\quad \beta_{2}$-agonist/long-acting muscarinic antagonist [ICS/LABA/LAMA]), (3) prescription of additional chronic therapy for COPD (theophylline or other methylxanthines, maintenance oral corticosteroids, phosphodiesterase- 4 inhibitors, macrolides, mucolytics or any other medication prescribed for COPD), or (4) respiratoryrelated death. An exacerbation is considered moderate/ severe exacerbation when the patient requires one of the following: (1) an acute oral corticosteroid course 
prescription, (2) antibiotics prescribed with evidence of a lower respiratory consultation at the same day, (3) respiratory-related hospital attendance or admission, or (4) respiratory-related accident and emergency/emergency room attendance. In the ASA group, the baseline from which time to treatment failure will be assessed for each patient, is defined as the day of indacaterol/glycopyrronium + add-on initiation following cluster-randomization of the site. The median time from cluster-randomization to indacaterol/glycopyrronium initiation (i.e. delay) across all ASA group sites will be determined. This delay value will be added to the time each control practice was clusterrandomized, to obtain the baseline timepoint for patients at control sites.

The secondary end points include assessment of proportion of patients with moderate/severe exacerbations, and total number of exacerbations at 12 months, based on electronic medical record data. Adherence will also be assessed, determined as Medication Possession Ratio (MPR) based on electronic medical record data as follows:

MPR $=($ Total pack days/observation period in days $) *$ 100, where,

Total Pack Days $=\sum$ (Number days per pack), and

Number days per pack $=$ Number of actuations per pack/Number of actuations per day.

The exploratory endpoints include assessment of costs from the OPCRD for (1) moderate/severe COPD exacerbations, (2) respiratory-related primary care consultations, (3) respiratory-related outpatient visits, (4) respiratoryrelated accident and emergency attendances, (5) respiratory-related hospital admissions, (6) inhalers prescribed, mean (SD) and numbers, categorized, (7) other COPDrelated drugs, (8) number of oral corticosteroid prescriptions, (9) number of acute antibiotic courses with evidence of a lower respiratory tract infection. The intervention package acceptance rate, defined as the percentage of patients who accepted the intervention package, of the total number of suitable patients at the ASA sites will also be assessed.

\section{Study Visits and Study Assessments}

Apart from the intervention initiation visit in the ASA practices, there will be no scheduled trial visits, to keep the trial closer to the normal clinical practice. All patient contacts and visits during the trial will be part of routine COPD management and care, in accordance with National Institute for Health and Care Excellence 2018 recommendations. $^{31}$
Suitable patients in the ASA group will be seen for a standard COPD review after the practice has been randomized. At this visit, if the patient is initiated on indacaterol/glycopyrronium from a different medication inhaler, they will receive indacaterol/glycopyrronium inhaler training. Patients already on indacaterol/glycopyrronium would have their inhaler technique checked as per standard COPD review. If the patient accepts the add-on device and app, the site practitioner/nurse would assist the patient to download the app and register as a user, and to set up the add-on device with the inhaler. Patients will be invited to use the adherence support system at reviews of their COPD. The system consists of an inhaler sensor device which is attached to the dual bronchodilator indacaterol/ glycopyrronium Breezhaler ${ }^{\circledR}$ and connected to an app on the patient's mobile phone. The sensor was developed and manufactured by Propeller Health and is small and unobtrusive. $^{20}$ The patient will be instructed on the use of the add-on device and the app and be trained on the functionalities of the technology (dose reminders, checking inhaler use statistics, recording COPD control by completing the COPD Assessment Test and receiving weekly COPD digests [can be through email]). Patients will also be instructed on the maintenance of the add-on device. Suitable patients in the control group will be invited for a standard COPD review at 12 months after randomization that will include completing an OPC COPD review questionnaire.

There will be no study-related assessments. All data are either routinely collected, and extracted from primary care records, or collected as part of COPD review. No additional assessments will be required to collect outcome data.

\section{Sample Size Considerations}

The sample size was calculated based on existing data from over 800 primary care practices within the Optimum Patient Care Research Database (OPCRD). ${ }^{32}$ Over 5555 patients in the OPCRD were identified as meeting the trial eligibility criteria. Of these, 3010 had outcome data available for 1 year and a further 2530 had a treatment failure within this outcome period.

For the primary endpoint, assuming that the median time to treatment failure in the control arm is 90 days as observed on the OPCRD and based on a clinically meaningful increase of $20 \%^{33}$ in the ASA (i.e. 108 days), we calculated that 82 practices per arm are required (total 164). This equates to a hazard in the control arm of 
0.007 per day and 0.0064 in the ASA, giving a hazard ratio of 0.83 . Assuming that over $50 \%$ of suitable patients accept the add-on device and app technology (on average, 17 suitable patients will be identified at 200 practices to qualify as suitable for the trial; of the suitable patients, it is estimated that an average of 8 will accept the technology), a total sample size of 1312 patients will provide $80 \%$ power, based on a $5 \%$ level of significance and an Intraclass Correlation Coefficient (ICC) of 0.038. To allow for a practice drop-out rate of approximately $6 \%$, 88 practices per arm will be enrolled. The ICC was calculated in the OPCRD dataset of 2530 patients from 508 practices, using a one-way random effects analysis of variance model, with the model fit in Stata V14 (Stata Corp, College Station, TX, USA) using the "loneway" command specifying "failure time" as the dependent variable, and practice ID as the independent variable. ${ }^{34}$

\section{Statistical Methods}

\section{Analysis Sets}

The analysis will be carried out in the primary trial population, which is defined to ensure population comparisons are scientifically valid in the ASA and control sites. The study includes primary population, secondary population and exploratory population, which is described in detail in Table 1 .

The following subgroups within each cluster of sites will be analyzed: (1) patients on the intervention package in the ASA sites and (2) subgroups of patients on indacaterol/glycopyrronium in the control sites and ASA sites.

For the primary analysis, time to treatment failure will be determined at the individual patient level, with standard errors adjusted for clustering of patients within the same practice. The primary comparison of trial arms will be based on the intention-to-treat principle including all patients identified as suitable and accepting of new technology. Additional details on the analysis, models and assumptions are described in Supplementary File S2.

The secondary endpoints will be analyzed in the primary population. For the adherence endpoint, we will use a linear mixed model to compare the mean medication possession ratio between the two arms. Random effects for the practice and fixed effects for the stratification factors used in the randomization algorithm will be included in the model. Level of significance will be set at $5 \%$ for both endpoints. Data will be analyzed in Stata V14 (Stata Corp, College Station, TX, USA). Details on exploratory and sensitivity analyses are described in Supplementary File S3.
Table I Definitions of Population Sets

\begin{tabular}{|l|l|}
\hline Population Set & Definition \\
\hline population & $\begin{array}{l}\text { ASA site: Group of patients who satisfy inclusion } \\
\text { criteria at baseline, and accepted the } \\
\text { intervention package } \\
\text { Control site: Group of patients who satisfy } \\
\text { inclusion criteria at baseline and are willing to } \\
\text { use intervention package (based on COPD } \\
\text { review questionnaire data collected at the end } \\
\text { of the study) }\end{array}$ \\
\hline Secondary & $\begin{array}{l}\text { ASA site: Group of patients satisfying selection } \\
\text { criteria (i.e. adherence to current mono/dual } \\
\text { therapy } \leq 50 \% \text { and who were offered the } \\
\text { intervention package (regardless of their } \\
\text { acceptance) } \\
\text { Control site: Group of patients satisfying } \\
\text { selection criteria (i.e. adherence to current } \\
\text { mono/dual therapy } \leq 50 \% \text { and continuing with } \\
\text { routine clinical care (LABA/LAMA, LABA, } \\
\text { LAMA, LABA/ICS, LABA/LAMA/ICS) }\end{array}$ \\
\hline Exploratory \\
population & $\begin{array}{l}\text { ASA site: Group of patients satisfying selection } \\
\text { criteria (i.e. adherence to current mono/dual } \\
\text { therapy } \leq 50 \% \text { ) and who accept or do not accept } \\
\text { the technology }\end{array}$ \\
\hline
\end{tabular}

Abbreviations: ASA, adherence support arm; COPD, chronic obstructive pulmonary disease; ICS, inhaled corticosteroids; LABA, long-acting beta-2 agonists; LAMA, long-acting muscarinic antagonists.

\section{Ethics Approval}

MAGNIFY trial protocol received approval from the East Midlands - Derby Research Ethics Committee on November 6, 2019 (REC ref: 19/EM/0238, IRAS: 260690) and from the UK Health Research Authority (December 2, 2019). OPCRD is approved by the Health Research Authority for clinical research use and governed by the Anonymized Data Ethics \& Protocol Transparency (ADEPT) Committee (ADEPT0719). The study protocol has also been registered with the European Union electronic Register of Post-Authorization Studies (EUPAS Register number EUPAS31405). Further details are described in the Supplementary File S4. The trial will be conducted in accordance with any applicable laws and regulations in the UK, and with the Declaration of Helsinki.

\section{Sources of Bias}

Pragmatic, cluster randomized trials are more exposed to biases. Therefore, we have adopted strategies to reduce the bias. $^{26,35}$ The potential sources of bias for MAGNIFY 
Table 2 Potential Sources of Bias and Measures to Address Bias

\begin{tabular}{|c|c|}
\hline Potential Source of Bias & Measures Taken to Address Bias \\
\hline $\begin{array}{l}\text { Index date } \\
\text { Date of initiation on the adherence package is used as the index date in } \\
\text { the ASA patients, but there is no equivalent index date in the control } \\
\text { arm. The median time from cluster randomization to the package } \\
\text { initiation (i.e. delay) across all adherence support group sites will be } \\
\text { determined and this delay value will be added to the time each control } \\
\text { practice was cluster randomized, to obtain the follow-up period start } \\
\text { date ("index date") for patients at control sites. This approach ensures } \\
\text { that the baseline is time-matched between the two arms, but may cause } \\
\text { bias because in the control arm the index date is not defined on } \\
\text { a patient-level as it is in the ASA }\end{array}$ & $\begin{array}{l}\text { Sensitivity analyses will be conducted using: } \\
\text { (a) the first COPD maintenance treatment after practice randomization } \\
\text { as the index date in the control arm, and } \\
\text { (b) the first primary care practitioner consultation after practice } \\
\text { randomization as index dates in the control arm }\end{array}$ \\
\hline $\begin{array}{l}\text { Treatment failures } \\
\text { Stepping up maintenance therapy is often considered as a treatment } \\
\text { failure in clinical studies. However, if any maintenance therapy step up } \\
\text { was considered a treatment failure, there would be potential bias for } \\
\text { more treatment failures in the control arm due to patients on } \\
\text { monotherapy being stepped up to LABA/LAMA or ICS/LABA (both in } \\
\text { line with current treatment recommendations), while in the ASA } \\
\text { initiation on the package (LABA/LAMA) at baseline of a patient } \\
\text { previously on monotherapy cannot be considered a treatment failure }\end{array}$ & $\begin{array}{l}\text { Only step up to triple therapy will be considered treatment failure in the } \\
\text { primary analysis. However, a sensitivity analysis will be conducted using } \\
\text { a modified definition of the primary outcome where any step up is } \\
\text { considered a treatment failure }\end{array}$ \\
\hline $\begin{array}{l}\text { Impact on prescribing decisions } \\
\text { Participation in the trial may drive LABA/LAMA prescribing decisions at } \\
\text { participating sites }\end{array}$ & $\begin{array}{l}\text { Only sites that are actively prescribing LABA/LAMA will be included in } \\
\text { the trial. LABA/LAMA is recommended for patients with COPD by the } \\
\text { current treatment guidelines }\end{array}$ \\
\hline
\end{tabular}

Abbreviations: ASA, adherence support arm; COPD, chronic obstructive pulmonary disease; ICS, inhaled corticosteroids; LABA, long-acting beta-2 agonists; LAMA, longacting muscarinic antagonists.

include index date, treatment failures and impact on prescribing decisions. The description for the sources and the measures taken to address these, are described in Table 2.

\section{Discussion}

Medication non-adherence is a global, complex and multifactorial challenge. ${ }^{36}$ Health interventions that aim at improving it are often not properly evaluated. Considering this, we have designed a pragmatic cluster randomized trial, MAGNIFY, to investigate the impact of a technology-based medication adherence approach in exacerbating COPD patients with poor adherence to mono/dual therapy.

The MAGNIFY study offers opportunities to capture patient medication adherence details in a real-life scenario. Cluster randomized trials generally include a broader range of patients and trial sites (by including communitybased, non-academic sites), and can evaluate outcomes that are aligned with the evidence needs of decisionmakers (e.g. patients, clinicians, payers, and policymakers). ${ }^{37}$ These trials are well-suited for providing evidence on effectiveness of clinical interventions, such as electronic monitoring, in real-world practice. ${ }^{38}$ Notably, they minimize the risk of cross contamination between intervention and control group patients within the same practice. Pragmatic study design sets the study in real clinical practice as far as possible. With the widespread use of electronic health records, there are increasing opportunities to integrate pragmatic cluster trial designs with real-world datasets to improve patient safety, data accuracy, and clinical trial efficiency.

Various approaches are being considered to monitor medication adherence because improving adherence to therapy has shown substantial health and economic benefits. ${ }^{39}$ A personalized treatment approach aided with digital inhaler technologies is one such approach. ${ }^{19,40}$ Technology-enabled inhalers offer a range of support such as patient reminders, feedback on inhaler technique and timing, and sensors to analyze inspiratory flow rates, and characterize inhaler technique. ${ }^{19,40}$ Another novel technology-driven approach to improve adherence and 
patient behavior is by using a smartphone app. ${ }^{41}$ The advantage with this approach is that it is constantly accessible, involves and educates the patient, and provides a repository for patient- and medication-specific information. A smartphone medication adherence-oriented app (adherence apps) can potentially consolidate all of the user's medication-specific information and thereby provide a more streamlined process to educate the individual about their disease. ${ }^{41}$ Compared with traditional approaches, technology-based approaches for medication adherence can reduce the cost and effort from the patient and caregiver's perspective. Additionally, the accuracy of the adherence measure, which is of great importance from the healthcare provider's point of view, can be enhanced using electronic-based systems. ${ }^{39}$ Therefore, in MAGNIFY, using an intervention package, a dual bronchodilator with an add-on inhaler sensor device and connected mobile app, offers enhanced adherence support benefits to patients with COPD in managing exacerbations.

Following the CONSORT statement guidelines for pragmatic cluster randomized trials, we ensured that the risk of contamination as a result of selection bias with preference of patient or physician will be minimized. ${ }^{27}$ The correct intervention according to COPD treatment guidelines for these patients is to address adherence and inhaler technique, not to step up therapy. ${ }^{18}$

\section{Conclusion}

We have described in detail the novel study design of the MAGNIFY trial. Positive outcomes from this pragmatic cluster randomized trial will provide real-life evidence on whether usage of technology-aided adherence support in combination with indacaterol/glycopyrronium Breezhaler ${ }^{\circledR}$ can be beneficial in patients with COPD, and whether it is cost-effective in usual practice. The results from this study will inform future approaches to improve adherence, offering the potential to improve patient outcomes and reduce healthcare costs.

\section{Abbreviations}

ASA, adherence support arm; COPD, chronic obstructive pulmonary disease; CE, Conformité Européenne; EMR, electronic medical record; $\mathrm{FEV}_{1}$, forced expiratory volume in 1 second; GOLD, Global Initiative for Chronic Obstructive Lung Disease; ICC, Intraclass Correlation Coefficient; ICS, inhaled corticosteroids; LAMA, longacting muscarinic antagonists; LABA, long-acting $\beta_{2}$ agonists; MPR, medication possession ratio; OPCRD,
Optimum Patient Care Research Database; OPC, Optimum Patient Care; RCTs, randomized clinical trials; TAP, technology adherence package.

\section{Data Sharing Statement}

Observational Pragmatic Research Institute (OPRI) will not provide access to patient-level data, if there is a reasonable likelihood that individual patients could be re-identified. In addition, clinical data, in some cases, have been collected subject to contractual or consent provisions that prohibit transfer to third parties. Such restrictions may preclude granting access under these provisions. Where co-development and data sharing agreements or other legal restrictions prevent companies from sharing data, companies will work with qualified requestors to provide summary information where possible.

\section{Acknowledgments}

The authors thank Mohammed Najeeb Ashraf, Phani Dantu and Rahul Lad (Novartis Healthcare Pvt. Ltd India) for providing medical writing support/editorial support, which was funded by Novartis, in accordance with Good Publication Practice (GPP3) guidelines (http://www. ismpp.org/gpp3).

\section{Author Contributions}

All authors made substantial contributions to conception and design, acquisition of data, or analysis and interpretation of data; took part in drafting the article or revising it critically for important intellectual content; agreed to submit to the current journal; gave final approval of the version to be published; and agree to be accountable for all aspects of the work.

\section{Funding}

The trial is sponsored by Observational Pragmatic Research International (OPRI) and receives co-funding from Novartis Pharma AG and OPRI. The add-on devices are provided by Propeller Health.

\section{Disclosure}

David Price has board membership with Amgen, AstraZeneca, Boehringer Ingelheim, Chiesi, Circassia, Mylan, Mundipharma, Novartis, Regeneron Pharmaceuticals, Sanofi Genzyme, Teva Pharmaceuticals, Thermofisher; consultancy agreements with Amgen, AstraZeneca, Boehringer Ingelheim, Chiesi, GlaxoSmithKline, Mylan, Mundipharma, Novartis, 
Pfizer, Teva Pharmaceuticals, Theravance; grants and unrestricted funding for investigator-initiated studies (conducted through Observational and Pragmatic Research Institute Pte Ltd) from AstraZeneca, Boehringer Ingelheim, Chiesi, Circassia, Mylan, Merck, Zentiva (Sanofi Generics), Mundipharma, Novartis, Pfizer, Regeneron Pharmaceuticals, Respiratory Effectiveness Group, Sanofi Genzyme, Teva Pharmaceuticals, Theravance, UK National Health Service; payment for lectures/speaking engagements from AstraZeneca, Boehringer Ingelheim, Chiesi, Cipla, GlaxoSmithKline, Kyorin, Mylan, Mundipharma, Novartis, Regeneron Pharmaceuticals, Sanofi Genzyme, Teva Pharmaceuticals; payment for the development of educational materials from Mundipharma, Novartis; payment for travel/ accommodation/meeting expenses from AstraZeneca, Boehringer Ingelheim, Mundipharma, Mylan, Novartis, Thermofisher; funding for patient enrolment or completion of research from Novartis; stock/stock options from AKL Research and Development Ltd which produces phytopharmaceuticals; owns $74 \%$ of the social enterprise Optimum Patient Care Ltd (Australia and UK) and 74\% of Observational and Pragmatic Research Institute Pte Ltd (Singapore); 92.61\% of Observational and Pragmatic Research Institute Pte Ltd (Singapore); 5\% shareholding in Timestamp which develops adherence monitoring technology; is peer reviewer for grant committees of the Efficacy and Mechanism Evaluation programme, and Health Technology Assessment; personal fees from Airway Vista Secretariat, EPG Communication Holdings Ltd, FIECON Ltd, Fieldwork International, OM Pharma SA, PeerVoice, Phadia AB, Spirosure Inc, Strategic North Limited, Synapse Research Management Partners S.L, Talos Health Solutions, WebMD Global LLC, outside the submitted work; and was an expert witness for GlaxoSmithKline.

Rupert Jones declares grants from Astra Zeneca, GlaxoSmithKline and Novartis and personal fees for consultancy, speaker's fees or travel support from Astra Zeneca, Boehringer Ingelheim, Glaxo Smith Kline, Novartis, Nutricia and OPRI.

Victoria Carter is an employee of the study sponsor Observational \& Pragmatic Research International Ltd (OPRI), the study sponsor, which has conducted paid research in respiratory disease on behalf of the following other organizations in the past 5 years: Aerocrine, AKL Ltd, Almirall, AstraZeneca, Boehringer Ingelheim, Chiesi, GlaxoSmithKline, Meda, Mundipharma, Napp, Novartis, Orion, Sanofi, Takeda and Teva.
Alan Kaplan received fee for advisory board or speakers bureau for Astra Zeneca, Boehringer Ingelheim, Covis, GSK, Merck Frosst, Pfizer, Purdue, Novartis, Sanofi, Teva, Trudel.

David MG Halpin has received personal fees from Aerogen, AstraZeneca, Boehringer Ingelheim, Chiesi, GSK, Novartis, Pfizer and Sanofi, and non-financial support from Boehringer Ingelheim and Novartis.

Anu Kemppinen and Allan Clark have nothing to disclose.

Hilary Pinnock was paid an honorarium for writing an on-line piece on supported self-management for Teva website. She is involved with several professional or educational organisations which receive multi-company sponsorship.

Job van Boven receives funding from the European Commission to Chair the European Network to Advance Best practices \& technoLogy on medication adherencE (COST Action CA19132, ENABLE). His institution (Medication Adherence Expertise Center of the northern Netherlands, MAECON, University Medical Center Groningen, Groningen, the Netherlands) has received consultancy fees, speaking fees, and/or research grants from AstraZeneca, Boehringer Ingelheim, Chiesi, eLucid mHealth, Menarini, Novartis, Teva and Trudell Medical related to medication adherence but all unrelated to this study.

Kai M. Beeh declares personal or institutional compensations from the following in the past 5 years: Kai Beeh is a full time employee of insaf Respiratory Research Institute. The institution has received compensation for services on advisory boards or consulting for AstraZeneca, Berlin Chemie, Boehringer, Chiesi, Cytos, GSK, Mundipharma, Novartis, Pohl Boskamp, Sanofi, Zentiva. The institution has received compensation for speaker activities in scientific meetings supported by AstraZeneca, Berlin Chemie, Boehringer, ERT, GSK, Novartis, Pfizer, Pohl Boskamp, Sanofi, Takeda. The institution has further received compensation for design and performance of clinical trials from AstraZeneca, Boehringer, GSK, Infinity, Mundipharma, Novartis, Parexel, Pearl Therapeutics, Pfizer, Revotar, Teva, Sterna, and Zentiva.

Konstantinos Kostikas was an employee and shareholder of Novartis Pharma AG until October 31, 2018 He have received honoraria for presentations and consultancy fees from AstraZeneca, Boehringer Ingelheim, Chiesi, ELPEN, GSK, Menarini, Novartis and Sanofi; his 
department has received funding and grants from AstraZeneca, Boehringer Ingelheim, Chiesi, Innovis, ELPEN, GSK, Menarini, Novartis and NuvoAir.

Nicolas Roche reports research grants from Boehringer Ingelheim, Novartis, Pfizer and personal fees for consultancy, advisory boards, lectures and education, participation to steering committees from Boehringer Ingelheim, Novartis, Pfizer, Teva, GSK, AstraZeneca, Chiesi, Sanofi, Trudell, Zambon.

James Chalmers reports grants and personal fees from AstraZeneca, grants and personal fees from Boehringer Ingelheim, personal fees from Chiesi, grants and personal fees from Gilead Sciences, grants and personal fees from GlaxoSmithKline, grants and personal fees from Novartis, personal fees from Zambon, from null, outside the submitted work; Dr. Chalmers reports grants and personal fees from AstraZeneca, grants and personal fees from Boehringer Ingelheim and Insmed, personal fees from Chiesi, grants and personal fees from Gilead Sciences, grants and personal fees from GlaxoSmithKline, grants and personal fees from Novartis, personal fees from Zambon, personal fees from Janssen, outside the submitted work.

Omar Usmani reports grants and personal fees from AstraZeneca, grants and personal fees from Boehringer Ingelheim, grants and personal fees from Chiesi, grants and personal fees from GlaxoSmithKline, personal fees from Napp, personal fees from Mundi Pharma, personal fees from Sandoz, personal fees from Takeda, grants from Edmond pharma, personal fees from Cipla, personal fees from Covis, personal fees from Novartis, personal fees from Mereo BioPharma, personal fees from Orion, personal fees from Menarini, personal fees from Deva, personal fees from Roche, personal fees from, Trudel, personal fees from UCB outside the submitted work.

Björn Holzhauer, Pascal Pfister, Hui Cao and Paul Mastoridis are employees of Novartis and holds stock in Novartis Pharma AG. The authors report no other conflicts of interest in this work.

\section{References}

1. GBD 2015 Mortality and Causes of Death Collaborators. Global, regional, and national life expectancy, all-cause mortality, and causespecific mortality for 249 causes of death, 1980-2015: a systematic analysis for the Global Burden of Disease Study 2015. Lancet. 2016;388:1459-1544.

2. Halpin DM, Miravitlles M, Metzdorf N, Celli B. Impact and prevention of severe exacerbations of COPD: a review of the evidence. Int J Chron Obstruct Pulmon Dis. 2017;12:2891-2908. doi:10.2147/ COPD.S139470
3. Andreas $\mathrm{S}$, Taube C. Inhaled therapy reduces COPD mortality. ERJ Open Res. 2020;6(4):00634-2020. doi:10.1183/23120541.006342020

4. Cecere LM, Slatore CG, Uman JE, et al. Adherence to long-acting inhaled therapies among patients with chronic obstructive pulmonary disease (COPD). COPD. 2012;9(3):251-258. doi:10.3109/ 15412555.2011.650241

5. van Boven JF, Chavannes NH, van der Molen T, Rutten-van Molken MP, Postma MJ, Vegter S. Clinical and economic impact of non-adherence in COPD: a systematic review. Respir Med. 2014;108 (1):103-113. doi:10.1016/j.rmed.2013.08.044

6. Ingebrigtsen TS, Marott JL, Nordestgaard BG, et al. Low use and adherence to maintenance medication in chronic obstructive pulmonary disease in the general population. J Gen Intern Med. 2015;30 (1):51-59. doi:10.1007/s11606-014-3029-0

7. Simoni-Wastila L, Wei YJ, Qian J, et al. Association of chronic obstructive pulmonary disease maintenance medication adherence with all-cause hospitalization and spending in a Medicare population. Am J Geriatr Pharmacother. 2012;10(3):201-210. doi:10.1016/j.amjopharm.2012.04.002

8. Agh T, Inotai A, Meszaros A. Factors associated with medication adherence in patients with chronic obstructive pulmonary disease. Respiration. 2011;82(4):328-334. doi:10.1159/000324453

9. George J, Kong DCM, Thoman R, et al. Factors associated with medication non adherence in patients with COPD. Chest. 2005;128 (5):3198-3204. doi:10.1378/chest.128.5.3198

10. Laforest L, Denis F, Van Ganse E, et al. Correlates of adherence to respiratory drugs in COPD patients. Prim Care Respir J. 2010;19 (2):148-154. doi:10.4104/pcrj.2010.00004

11. Wei L, Yang X, Li J, et al. Effect of pharmaceutical care on medication adherence and hospital admission in patients with chronic obstructive pulmonary disease (COPD): a randomized controlled trial. J Thorac Dis. 2014;6(6):656-662. doi:10.3978/j.issn.20721439.2014.06.20

12. Khdour MR, Hawwa AF, Kidney JC, et al. Potential risk factors for medication non-adherence in patients with chronic obstructive pulmonary disease (COPD). Eur J Clin Pharmacol. 2012;68 (10):1365-1373. doi:10.1007/s00228-012-1279-5

13. Krauskopf K, Federman AD, Kale MS, et al. Chronic obstructive pulmonary disease illness and medication beliefs are associated with medication adherence. COPD. 2015;12(2):151-164. doi:10.3109/ 15412555.2014.922067

14. Bosnic-Anticevich S, Chrystyn H, Costello RW, et al. The use of multiple respiratory inhalers requiring different inhalation techniques has an adverse effect on COPD outcomes. Int $J$ Chron Obstruct Pulmon Dis. 2016;12:59-71. doi:10.2147/COPD.S117196

15. Melani AS, Canessa P, Coloretti I, et al. Inhaler mishandling is very common in patients with chronic airflow obstruction and long-term home nebuliser use. Respir Med. 2012;106(5):668-676. doi:10.1016/ j.rmed.2011.11.016

16. Claudia G, Dieterle T, Breitenstein A-L, et al. Use and inhalation technique of inhaled medication in patients with asthma and COPD: data from a randomized controlled trial. Respir Res. 2018;19 (237):1-15. doi:10.1186/s12931-017-0698-3

17. Braido F, Chrystyn H, Baiardini I, et al. "Trying, but failing" - the role of inhaler technique and mode of delivery in respiratory medication adherence. J Allergy Clin Immunol Pract. 2016;4(5):823-832. doi:10.1016/j.jaip.2016.03.002

18. Global strategy for the diagnosis, management, and prevention of chronic obstructive pulmonary disease; 2020. Report. Available from: www.goldcopd.org. Accessed June 6, 2020.

19. Garrett G, Costello RW. Personalizing medicine - could the smart inhaler revolutionize treatment for COPD and asthma patients? Expert Opin Drug Deliv. 2019;16(7):675-677. doi:10.1080/ 17425247.2019.1628017 
20. Chen J, Kaye L, Tuffli M, et al. Passive monitoring of short-acting beta-agonist use via digital platform in patients with chronic obstructive pulmonary disease: quality improvement retrospective analysis. JMIR Form Res. 2019;3(4):e13286. doi:10.2196/13286

21. Moore A, Preece A, Sharma R, et al. A randomised controlled trial of the effect of a connected inhaler system on medication adherence in uncontrolled asthmatic patients. Eur Respir J. 2020:2003103. doi:10.1183/13993003.03103-2020

22. Gossec L, Tubach F, Dougados M, Ravaud P. Reporting of adherence to medication in recent randomized controlled trials of 6 chronic diseases: a systematic literature review. Am J Med Sci. 2007;334 (4):248-254. doi:10.1097/MAJ.0b013e318068dde8

23. Roche N, Anzueto A, Bosnic Anticevich S, et al. The importance of real-life research in respiratory medicine: manifesto of the Respiratory Effectiveness Group. Eur Respir J. 2019;54 (3):1901511. doi:10.1183/13993003.01511-2019

24. Vestbo J, Anderson JA, Calverley PMA, et al. Adherence to inhaled therapy, mortality and hospital admission in COPD. Thorax. 2009;64 (11):939-943. doi:10.1136/thx.2009.113662

25. Dal-Ré R, Janiaud P, Ioannidis JP. Real-world evidence: how pragmatic are randomized controlled trials labeled as pragmatic? BMC Med. 2018;16(1):49. doi:10.1186/s12916-018-1038-2

26. Gamerman V, Cai T, Elsäßer A. Pragmatic randomized clinical trials: best practices and statistical guidance. Health Serv Outcomes Res Methodol. 2019;19(1):23-35. doi:10.1007/s10742-018-0192-5

27. Campbell MK, Elbourne DR, Altman DG. CONSORT statement: extension to cluster randomised trials. BMJ. 2004;328 (7441):702-708. doi:10.1136/bmj.328.7441.702

28. Zwarenstein M, Treweek S, Gagnier JJ, et al. Pragmatic trials in healthcare: improving the reporting of pragmatic trials: an extension of the CONSORT statement. BMJ. 2008;337(nov11 2):a2390. doi:10.1136/bmj.a2390

29. Alexander E, Seyi S, Brooklyn S, et al. Strategies that promote sustainability in quality improvement activities for chronic disease management in healthcare settings: a practical perspective. Qual Prim Care. 2020;28(6):55-60.

30. National General Practice Profiles. Public health England. Available from: https://fingertips.phe.org.uk/profile/general-practice. Accessed November 24, 2019.
31. National Institute for Health and Care Excellence. Chronic obstructive pulmonary disease in over 16s: diagnosis and management. Clinical guideline; December 2018. Available from: https://www. nice.org.uk/guidance/ng115. Accessed November 24, 2019.

32. NHS Health Research Authority. Optimum patient care research database. Available from: https://www.hra.nhs.uk/planning-andimproving-research/application-summaries/research-summaries/opti mum-patient-care-research-database/. Accessed November 24, 2019.

33. Chapman KR, Bergeron $\mathrm{C}$, Bhutani $\mathrm{M}$, et al. Do we know the minimal clinically important difference (MCID) for COPD exacerbations? COPD. 2013;10(2):243-249. doi:10.3109/ 15412555.2012.733463

34. Donner AA. Review of inference procedures for the intraclass correlation coefficient in the one-way random effects model. Int Stat Rev. 1986;54(1):67-82. doi:10.2307/1403259

35. Ernst E, Canter PH. Limitations of "pragmatic" trials. Postgrad Med J. 2005;81(954):203. doi:10.1136/pgmj.2004.026807

36. Meichembaum D, Turk D. Facilitating Treatment Adherence: A Practitioner's Guidebook. New York: Plenum; 1987.

37. Murphy AW, Esterman A, Pilotto LS. Cluster randomized controlled trials in primary care: an introduction. Eur J Gen Pract. 2006;12 (2):70-73. doi:10.1080/13814780600780627

38. Law LM, Wason JM. Design of telehealth trials-introducing adaptive approaches. Int J Med Inform. 2014;83(12):870-880. doi:10.1016/j. ijmedinf.2014.09.002

39. van Boven JF, Tommelein E, Boussery K, et al. Improving inhaler adherence in patients with chronic obstructive pulmonary disease: a cost-effectiveness analysis. Respir Res. 2014;15(1):66. doi:10.1186/ 1465-9921-15-66

40. Blakey JD, Bender BG, Dima AL, et al. Digital technologies and adherence in respiratory diseases: the road ahead. Eur Respir J. 2018;52(5):1801147. doi:10.1183/13993003.01147-2018

41. Dayer L, Heldenbrand S, Anderson P, Gubbins PO, Martin BC. Smartphone medication adherence apps: potential benefits to patients and providers. J Am Pharm Assoc. 2013;53(2):172-181. doi:10.1331/ JAPhA.2013.12202
Pragmatic and Observational Research

\section{Publish your work in this journal}

Pragmatic and Observational Research is an international, peerreviewed, open access journal that publishes data from studies designed to reflect more closely medical interventions in realworld clinical practice compared with classical randomized controlled trials (RCTs). The manuscript management system is completely online and includes a very quick and fair peer-review system. Visit http://www.dovepress.com/testimonials.php to read real quotes from published authors. 